\title{
Individuals requiring orthognathic surgery have more depression and pain than controls
}

\author{
Aline Monise SEBASTIANI(a) \\ Jennifer Tsi GERBER(b) \\ Isabela Polesi BERGAMASCHI(a) \\ Maria Fernanda PETINATI(b) \\ Michelle Nascimento MEGER ${ }^{(b)}$ \\ Delson João da COSTA ${ }^{(a)}$ \\ João Armando BRANCHER(b) (D) \\ Erika Calvano KÜCHLER(c) \\ Rafaela SCARIOT(a) Department of Stomatology, Curitiba, PR, Brazil. \\ (a) Universidade Federal do Paraná - UFPR, \\ (b) Universidade Positivo, School of Health and \\ Biological Sciences, Curitiba, PR, Brazil. \\ (c) Universidade de São Paulo - USP, Ribeirão \\ Preto Dental School, Department of \\ Pediatric Dentistry, Ribeirão Preto, SP, Brazil.
}

Declaration of Interests: The authors certify that they have no commercial or associative interest that represents a conflict of interest in connection with the manuscript.

\section{Corresponding Author:}

Aline Sebastiani

E-mail: sebastiani.aline@gmail.com

htrps://doi.org/10.1590/1807-3107bor-2021.vol35.0091

Submitted: January 31, 2020

Accepted for publication: October 3, 2020

Last revision: January 10, 2021

\begin{abstract}
The objective of this study was to evaluate if individuals with dentofacial deformities (DFD) who require orthognathic surgery are affected more by depression and pain. A case-control study was performed with 195 individuals. In the DFD group, 145 individuals with Class II and III malocclusion requiring orthognathic surgery were selected. The control group was composed of 50 individuals with no DFD. All patients were diagnosed according to the Research Diagnostic Criteria for Temporomandibular Disorders (RDC/TMD). Data were analyzed with a significance level of 0.05 . The DFD group more often presented severe depression $(p=0.020)$ and chronic pain $(p=0.017)$. They also presented higher prevalence of Nonspecific Physical Symptoms Including Pain $(p=0.002)$ and Nonspecific Physical Symptoms Excluding Pain $(p=0.002)$. Concerning TMD symptoms, the DFD group had more myofascial $(p=0.002)$ and articular pain $(p=0.041)$. Therefore, the results of this study suggest that depression and pain are more common in individuals with DFD requiring orthognathic surgery compared with individuals without DFD.
\end{abstract}

Keywords: Orthognathic Surgery; Chronic Pain; Depression;

Temporomandibular Joint Disorders.

\section{Introduction}

Individuals with dentofacial deformities (DFD), especially those that require orthognathic surgery, commonly present psychological alterations ${ }^{1}$ due to esthetic impairment, functional problems, orofacial pain, and chronic headaches, ${ }^{2}$ which may influence their self-esteem and personal relationships owing to difficulty in establishing effective social ties. ${ }^{1}$

Normally, during orthognathic surgery planning, surgeons give more importance to the skeletal alterations and functional and esthetics parameters than to psychological aspects. However, in order to achieve a successful outcome, patients should be treated as a whole. Therefore, it is fundamental that surgeons understand all the physical and psychological symptoms associated with patients requiring orthognathic surgery, since the majority of them experience a negative influence of their appearance on their psychosocial well-being. ${ }^{3}$ 
Individuals with DFD have a higher prevalence of depression than the general population, ${ }^{1}$ and depression is a major predictor of a poorer quality of life. However, orthognathic surgery seems to improve these conditions. ${ }^{4}$ Depression is a complex disorder manifested in many different ways. ${ }^{5}$ The characteristics of people with depression include not only an increase in negative emotions such as hopelessness or anger, but also a relative depletion of positive effective resources, such as the ability to experience reward-related pleasure. A striking feature of depression is the collapse of the motivational drive to deal with challenges of life, leading to withdrawal and behavior inhibition. ${ }^{6}$ Biological pathways are also related with this condition, such as decreased level of endogenous dopaminergic release, which can significantly contribute to the pathology of depression. ${ }^{7}$

Depression seems to be more common in patients with DFD who also have temporomandibular disorders (TMD). ${ }^{8}$ Pain, especially chronic pain, makes lives unbearable for many people. ${ }^{9}$ Chronic pain can also result in a hypodopaminergic state, interfering with motivation. ${ }^{10}$

Thus, the aim of this study was to compare the level of depression and pain in individuals with DFD who require orthognathic surgery with that of a control group.

\section{Methodology}

\section{Study design and sample}

The research project was approved by the local Ethics in Research Committee protocol number: CAEE 69240817.7.0000.0093 and CAAE 69725317.5.0000.0102, and complies with the Declaration of Helsinki for studies involving human subjects.

A case-control study was performed with a total of 195 individuals composing the DFD group and the control group. The inclusion criteria were adult patients of both sexes under treatment at the Maxillofacial Service at Positivo University and Federal University of Parana. The DFD group was composed by 145 individuals previously diagnosed by orthodontists and referred for ortho-surgical treatment, who were consecutively select during a period of three years. They were evaluated by a group of senior surgeons who selected patients with Class II or III skeletal malocclusion according to facial analysis and clinical exam of the occlusion. For the control group, 50 individuals with no DFD were selected in the preoperative evaluation for dental extraction. The people in the control group were checked by the same group of surgeons to confirm that there was no skeletal discrepancy and that they presented a Class I molar relationship. The exclusion criteria for both groups were patients who had a previous history of temporomandibular joint (TMJ) surgery, any syndrome, diagnosis of neuronal diseases, dental inflammatory processes, and several missing teeth.

\section{Data collection}

Individual characteristics were collected like sex, age, and ethnicity. The self-reported ethnicity was dichotomized in Caucasian and non-Caucasian.

Both groups were evaluated with the Research Diagnostic Criteria for Temporomandibular DisordersRDC-TMD index. ${ }^{11}$

According to axis II of the RDC-TMD, the individuals were evaluated for Depression, Chronic Pain, Nonspecific Physical Symptoms Including Pain (NPSIP), and Nonspecific Physical Symptoms Excluding Pain (NPSEP). According to these criteria, chronic pain was classified in grades from 0 to IV, being: 0 - no limitation; I - low intensity, II - high intensity, III - moderate limitation, and IV - severe limitation. The variable was dichotomized into absence or presence of chronical pain, being absence of chronical pain individuals with grade 0 , and presence of chronical pain, individuals with grade I or higher. For the Depression variable, participants were classified into normal, moderate, or severe. The NPSIP and NPSEP variables were dichotomized into presence or absence of these conditions, being a normal diagnosis considered as "absence" and a moderate or severe diagnosis, "presence" of the condition. With respect to axis I, individuals were evaluated for the three different diagnoses of TMD, as having presence or absence of myofascial pain, disc displacement on at least one side, and TMJ painful conditions (arthralgia and osteoarthritis). 


\section{Statistical analysis}

The results were submitted to inferential analyses with a significance level of 0.05 using the Statistical Package for the Social Sciences software (SPSS, v. 24.0, IBM, city, USA). In order to verify the matching of groups, the Chi-square and Mann-Whitney tests were used. In the logistic regression model, the groups were compared with regard to all diagnoses of RDC-TMD. Adjustments by sex were performed because it is known that depression and pain symptoms are more common in women than in men $^{12,13}$. Odds ratio (OR) was calculated to quantify the strength of the association.

\section{Results}

The groups were similar with regard to sex and ethnicity $(p>0.05)$. The median age in the case group was 28.0 (16-66), and in the control group, 23.5 (18--61). These data are shown in Table 1. The statistical power varied from $65 \%$ to $92 \%$ for axis II outcome variables.

The DFD group was composed of 52 men and 93 women, and 48 and 97 presented Class II and Class III malocclusion, respectively. In the Class II group, 6 individuals presented asymmetry, 12 , vertical maxillary excess, and 3, open bite. In the Class III group, 33 had asymmetry, 5 had vertical maxillary excess, and 5 had open bite.

Table 2 compares the RDC-TMD diagnosis between groups. The individuals with DFD had 5.89 times

Table 1. Individual characteristics of individuals with dentofacial deformities (DFD) and control group.

\begin{tabular}{|c|c|c|c|}
\hline \multirow{2}{*}{ Diagnose } & DFD group & Control group & \multirow{2}{*}{$\mathrm{p}$-value } \\
\hline & n (\%) & n (\%) & \\
\hline \multicolumn{4}{|l|}{ Sex } \\
\hline Male & 52 (35.9) & $19(38.0)$ & \\
\hline Female & $93(64.1)$ & $31(62.0)$ & 0.564 \\
\hline \multicolumn{4}{|l|}{ Ethnicity } \\
\hline Caucasian & $115(79.3)$ & $43(86.0)$ & \\
\hline Non-caucasian & $29(20.7)$ & 7 (14.0) & 0.336 \\
\hline \multicolumn{4}{|l|}{ Age } \\
\hline Median (min-max) & $28.0(16-66)$ & $23.5(18-61)$ & 0.015 \\
\hline
\end{tabular}

Qui-square, and Mann Whitney, with confidence level of 0.05 . higher chance of having severe depression, 2.36 times higher chance to have chronic pain, 3.97 times higher chance to have NPSIP, and 4.01 times higher chance to have NPESP when compared to the control group. Besides, the DFD group had 7 times higher chance to have myofascial pain and 3.12 times higher chance to have articular pain $(\mathrm{p}<0.05)$.

\section{Discussion}

Our results demonstrated that emotional and physical problems were more prevalent in individuals with DFD who require orthognathic surgery when compared to the control group. Severe depression was one of the more substantial results obtained from our study, with five times more chance of occurrence in individuals who require orthognathic surgery. Moderate depression occurrence did not differ between groups. Our results agree with a study conducted by Yao et al. ${ }^{1}$ that also showed more depressive symptoms in patients requiring orthognathic surgery.

Although orthognathic surgery provides a moderate improvement in psychological and social quality of life in the patients, depression is a major predictor of poor quality of life in this population, and interferes significantly with the vitality, social aspects of the individual, and mental health after surgery. ${ }^{14}$ Thus, the importance of diagnosis and treatment of these individuals before surgery is demonstrated. Chronic pain and somatization (NPSIP and NPESP) were also higher in individuals with DFD. Zhang et al. ${ }^{15}$ showed significantly higher values of somatization, obsessive-compulsiveness, interpersonal sensitivity, depression, anxiety, and paranoid ideation in people with malocclusion compared to individuals with normal malocclusion.

Chances to have myofascial pain were seven times higher in cases compared to controls, which corroborates the results of other studies. ${ }^{16,17,18}$ The correlation between facial skeletal morphology and muscular functionality were previously studied ${ }^{19}$. The patients with skeletal malocclusion and DFD seemed to present muscular incoordination and instability. In a study by Di Palma et al., ${ }^{19}$ electromyographic evaluations demonstrated improvements in 
Table 2. Comparison of RDC/TMD diagnoses between individuals with dentofacial deformities (DFD) and control group.

\begin{tabular}{|c|c|c|c|c|}
\hline \multirow{2}{*}{ Diagnosis } & DFD group $(n=145)$ & Control group $(n=50)$ & \multirow{2}{*}{$p$-value ${ }^{a}$} & \multirow{2}{*}{$\mathrm{OR}^{a}(95 \% \mathrm{Cl})$} \\
\hline & n (\%) & n (\%) & & \\
\hline \multicolumn{5}{|l|}{ Depression } \\
\hline Normal & $93(65.0)$ & $40(80.0)$ & reference & reference \\
\hline Moderate & $25(17.0)$ & $8(16.0)$ & 0.481 & $1.39(0.56-3.43)$ \\
\hline Severe & $26(18.0)$ & $2(4.0)$ & 0.020 & $5.89(1.32-26.21)$ \\
\hline \multicolumn{5}{|l|}{ Chronic pain } \\
\hline Absence & $80(55.2)$ & $37(74.0)$ & reference & reference \\
\hline Presence & $65(44.8)$ & $13(26.0)$ & 0.017 & $2.36(1.17-4.78)$ \\
\hline \multicolumn{5}{|l|}{ NPSIP } \\
\hline Absence & $88(61.5)$ & $43(86.0)$ & reference & reference \\
\hline Presence & $55(38.5)$ & 7 (14.0) & 0.002 & $3.97(1.65-9.53)$ \\
\hline \multicolumn{5}{|l|}{ NPSEP } \\
\hline Absence & $88(61.5)$ & $43(86.0)$ & reference & reference \\
\hline Presence & $55(38.5)$ & $7(14.0)$ & 0.002 & $4.01(1.66-9.68)$ \\
\hline \multicolumn{5}{|c|}{ Miofascial pain } \\
\hline Absence & $101(69.6)$ & $47(94.0)$ & reference & reference \\
\hline Presence & $44(30.4)$ & $3(6.0)$ & 0.002 & $7.00(2.08-23.49)$ \\
\hline \multicolumn{5}{|c|}{ Disc displacement } \\
\hline Absence & $90(62.1)$ & $34(68.0)$ & reference & reference \\
\hline Presence & $55(37.9)$ & $16(32.0)$ & 0.467 & $1.29(0.65-2.57)$ \\
\hline \multicolumn{5}{|l|}{ TMJ pain } \\
\hline Absence & $114(78.6)$ & $46(92.0)$ & reference & reference \\
\hline Presence & $31(21.4)$ & $4(8.0)$ & 0.041 & $3.12(1.05-9.29)$ \\
\hline
\end{tabular}

Logistic regression adjusted by sex, with confidence level of 0.05. ORa: adjusted odds ratio; $\mathrm{Cl}$ : confidence level; NPSIP: nonspecific physical symptoms including pain; NPSEP: nonspecific physical symptoms excluding pain; TMJ: temporomandibular joint.

neuromuscular equilibrium after surgical intervention due to better occlusal stability, suggesting that severe malocclusion affects muscular activity. We also found an increased prevalence in TMJ pain in cases but disk displacements was similar between groups. Our results are similar to the study by Abrahamsson et al. ${ }^{16}$ that also found a higher prevalence of myofascial pain and arthralgia in patients requiring orthognathic surgery compared to the control group and did not find differences in disc displacements. Thus, skeletal malocclusions do not seem to cause structural changes in TMJ, however they are related to more severe psychological suffering and painful symptoms.

Chronic pain is one of the critical determining factors for depression, and their coexistence tends to further aggravate the severity of both disorders. ${ }^{20}$
According to Sheng et al., ${ }^{20}$ patients suffering from depression induced by chronic pain have worse prognosis than those suffering from chronic pain alone; and chronic pain and depression are closely correlated in terms of occurrence and development, and mutually promote their severity progression. Somatization is also associated with depression and an important predictor of altered central pain modulation in chronic musculoskeletal pain..$^{15}$ These findings from the literature explain the high occurrence of depression, somatization, and chronic pain in our study, and also the correlation between them, suggesting a high interdependence of these symptoms.

To date, the pathophysiological mechanisms of chronic pain and depression, and their mutual correlation, have not been identified. However, 
neuroplasticity changes induced by pain and depression and changes in the neurobiological mechanism have been revealed. Monoamine neurotransmitters have been studied with regard to the molecular mechanisms involved in chronic pain and depression. The classic hypothesis is that depression may occur as a result of decreased availability of serotonin and norepinephrine neurotransmitters in the central nervous system. Monoamine neurotransmitters are also vital in the occurrence and development of pain. ${ }^{20}$

A study by Mladenovic et al. ${ }^{17}$ found that myofascial pain was related to a higher level of depression and somatization in people with Class III malocclusions. In another study, it was found that depression is higher in patients requiring orthognathic surgery that also had TMD. ${ }^{8}$

The inflammatory process and consequent afferent activity might be intense enough to establish a central process..$^{21}$ Altered central pain modulation is the predominant pain mechanism in a proportion of chronic musculoskeletal pain disorders ${ }^{22}$. When TMD is chronic, especially with myofascial pain, it is considered a functional pain syndrome similar to fibromyalgia. ${ }^{23}$ Functional disorders tend to be related to central sensitization and amplified pain perception. ${ }^{24}$ The pathophysiological mechanisms of such pain conditions remain unknown. However, it is believed that amplified pain perception, alterations in brain activity, immune and neuroendocrine activities, and genetic predisposition may be involved..$^{23}$

Our study had some limitations, and future studies are necessary with more precise diagnosis of psychological alterations performed by a specialist. Also, more investigation is needed about predictive variables of depression and chronic pain occurrence, like aesthetic perception, type of DFD, and social behaviors. Additionally, a study with a follow-up after orthognathic surgery is necessary to verify how these conditions evolve after the treatment to correct the DFD.

\section{Conclusion}

Individuals with DFD who require orthognathic surgery have higher prevalence of depression, chronic pain, NPSIP, and NPSEP, compared to individuals without DFD. They also present more myofascial pain and articular pain.

\section{References}

1. Yao S, Zhou J, Li Z. Psychologic health status of patients undergoing orthognathic surgery. J Craniofac Surg. 2014 Nov;25(6):e540-3. https://doi.org/10.1097/SCS.0000000000001042

2. Agbaje J, Luyten J, Politis C. Pain complaints in patients undergoing orthognathic surgery. Pain Res Manag. 2018 Jul;2018:4235025. https://doi.org/10.1155/2018/4235025

3. Steenen SA, Teeseling RA, Vulink NC, Becking AG. [Psychological aspects of orthognathic surgery]. Ned Tijdschr Tandheelkd. 2014 Sep;121(9):446-52. https://doi.org/10.5177/ntvt.2014.09.14114

4. Brunault P, Battini J, Potard C, Jonas C, Zagala-Bouquillon B, Chabut A, et al. Orthognathic surgery improves quality of life and depression, but not anxiety, and patients with higher preoperative depression scores improve less. Int J Oral Maxillofac Surg. 2016 Jan;45(1):26-34. https://doi.org/10.1016/j.ijom.2015.07.020

5. Anthes E. Depression: a change of mind. Nature. 2014, 13;515(7526):185-7. https://doi.org/10.1038/515185a

6. Kasch KL, Rottenberg J, Arnow BA, Gotlib IH. Behavioral activation and inhibition systems and the severity and course of depression. J Abnorm Psychol. 2002 Nov;111(4):589-97. https://doi.org/10.1037/0021-843X.111.4.589

7. Pearson-Fuhrhop KM, Dunn EC, Mortero S, Devan WJ, Falcone GJ, Lee P, et al. Dopamine genetic risk score predicts depressive symptoms in healthy adults and adults with depression. PLoS One 2014, 16;9(5):e93772. https://doi.org/10.1371/journal.pone.0093772. eCollection 2014.

8. Mladenović I, Jović N, Čutović T, Mladenović G, Kozomara R. Temporomandibular disorders after orthognathic surgery in patients with mandibular prognathism with depression as a risk factor. Acta Odontol Scand. 2013 Jan;71(1):57-64. https://doi.org/10.3109/00016357.2011.654239

9. Elman I, Borsook D. Common brain mechanisms of chronic pain and addiction. Neuron. 2016 Jan;89(1):11-36. https://doi.org/10.1016/j.neuron.2015.11.027 
Individuals requiring orthognathic surgery have more depression and pain than controls

10. Taylor AM, Becker S, Schweinhardt P, Cahill C. Mesolimbic dopamine signaling in acute and chronic pain: implications for motivation, analgesia, and addiction. Pain. 2016 Jun;157(6):1194-8. https://doi.org/10.1097/i.pain.0000000000000494

11. Dworkin SF, LeResche L. Research diagnostic criteria for temporomandibular disorders: review, criteria, examinations and specifications, critique. J Craniomandib Disord. 1992;6(4):301-55.

12. Pieretti S, Di Giannuario A, Di Giovannandrea R, Marzoli F, Piccaro G, Minosi P, et al. Gender differences in pain and its relief. Ann Ist Super Sanita. 2016 Apr-Jun;52(2):184-9. https://doi.org/10.4415/ANN_16_02_09

13. Sramek JJ, Murphy MF, Cutler NR. Sex differences in the psychopharmacological treatment of depression. Dialogues Clin Neurosci. 2016 Dec;18(4):447-57. https://doi.org/10.31887/DCNS.2016.18.4/ncutler

14. de Ávila ED, de Molon RS, Loffredo LC, Massucato EM, Hochuli-Vieira E. Health-related quality of life and depression in patients with dentofacial deformity. Oral Maxillofac Surg. 2013 Sep;17(3):187-91. https://doi.org/10.1007/s10006-012-0338-5

15. Zhang L, Liu X, Zheng GJ, Zhou L, Lin DY, Wang XD, et al. Eysenck personality and psychosocial status of adult patients with malocclusion. Asian Pac J Trop Med. 2012 Feb;5(2):151-6. https://doi.org/10.1016/S1995-7645(12)60015-7

16. Abrahamsson C, Henrikson T, Nilner M, Sunzel B, Bondemark L, Ekberg EC. TMD before and after correction of dentofacial deformities by orthodontic and orthognathic treatment. Int J Oral Maxillofac Surg. 2013 Jun;42(6):752-8. https://doi.org/10.1016/j.ijom.2012.10.016

17. Mladenović I, Dodić S, Stošić S, Petrović D, Cutović T, Kozomara R. TMD in class III patients referred for orthognathic surgery: psychological and dentition-related aspects. J Craniomaxillofac Surg. 2014 Dec;42(8):1604-9. https://doi.org/10.1016/i.jcms.2014.04.029

18. Bertoli FMP, Bruzamolin CD, Kranz GOA, Losso EM, Brancher JA, Souza JF. Anxiety and malocclusion are associated with temporomandibular disorders in adolescents diagnosed by RDC/TMD. A cross-sectional study. J Oral Rehabil. 2018 Oct;45(10):747-55. https://doi.org/10.1111/joor.12684

19. Di Palma E, Gasparini G, Pelo S, Tartaglia GM, Chimenti C. Activities of masticatory muscles in patients after orthognathic surgery. J Craniomaxillofac Surg. 2009 Oct;37(7):417-20. https://doi.org/10.1016/i.jcms.2009.05.004

20. Sheng J, Liu S, Wang Y, Cui R, Zhang X. The Link between Depression and Chronic Pain: Neural Mechanisms in the Brain. Neural Plast. 2017;2017:9724371. https://doi.org/10.1155/2017/9724371

21. Merrill RL. Central mechanisms of orofacial pain. Dent Clin North Am. 2007 Jan;51(1):45-59. https://doi.org/10.1016/i.cden.2006.09.010

22. Clark J, Nijs J, Yeowell G, Goodwin PC. What are the predictors of altered central pain modulation in chronic musculoskeletal pain populations? A systematic review. Pain Physician. 2017 Sep;20(6):487-500.

23. Kim SE, Chang L. Overlap between functional GI disorders and other functional syndromes: what are the underlying mechanisms? Neurogastroenterol Motil. 2012 Oct;24(10):895-913. https://doi.org/10.1111/j.1365-2982.2012.01993.x

24. Lorduy KM, Liegey-Dougall A, Haggard R, Sanders CN, Gatchel RJ. The prevalence of comorbid symptoms of central sensitization syndrome among three different groups of temporomandibular disorder patients. Pain Pract. 2013 Nov;13(8):604-13. https://doi.org/10.1111/papr.12029 